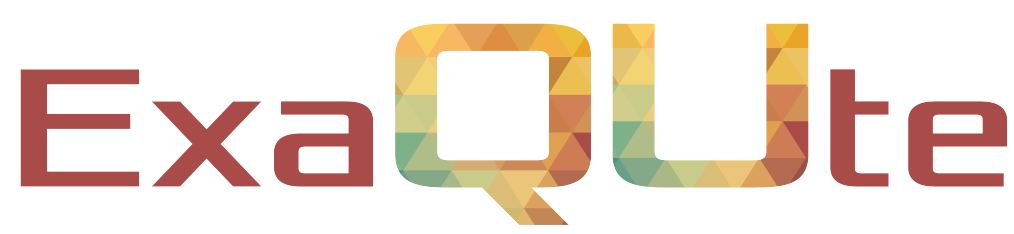

Exascale Quantification of Uncertainties for

Technology and Science Simulation

\title{
D1.2 First release of the softwares
}

\section{Document information table}

\begin{tabular}{|l|l|}
\hline Contract number: & 800898 \\
\hline Project acronym: & ExaQUte \\
\hline Project Coordinator: & CIMNE \\
\hline Document Responsible Partner: & CIMNE \\
\hline Deliverable Type: & OTHER: Software release \\
\hline Dissemination Level: & CO \\
\hline Status: & Final version \\
\hline
\end{tabular}

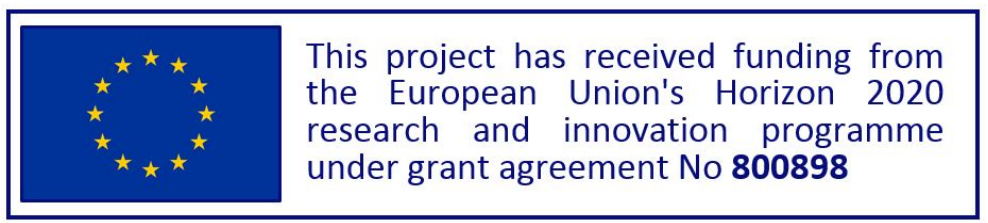




\section{Authoring}

\begin{tabular}{|l|l|l|l|l|}
\hline Prepared by: & Partner & Modified Page/Sections & Version & Comments \\
\hline Authors & & & & \\
\hline Riccardo Tosi & CIMNE & & & \\
\hline & & & & \\
\hline Contributors & & & & \\
\hline Ramon Amela & BSC & & & \\
\hline Marc Nuñez & CIMNE & & & \\
\hline Carlos Roig & CIMNE & & & \\
\hline Rosa M. Badia & BSC & & & \\
\hline Riccardo Rossi & CIMNE & & & \\
\hline & & & & \\
\hline
\end{tabular}

\section{Change Log}

\section{\begin{tabular}{|l|l|l|}
\hline Versions & Modified Page/Sections & Comments
\end{tabular}}
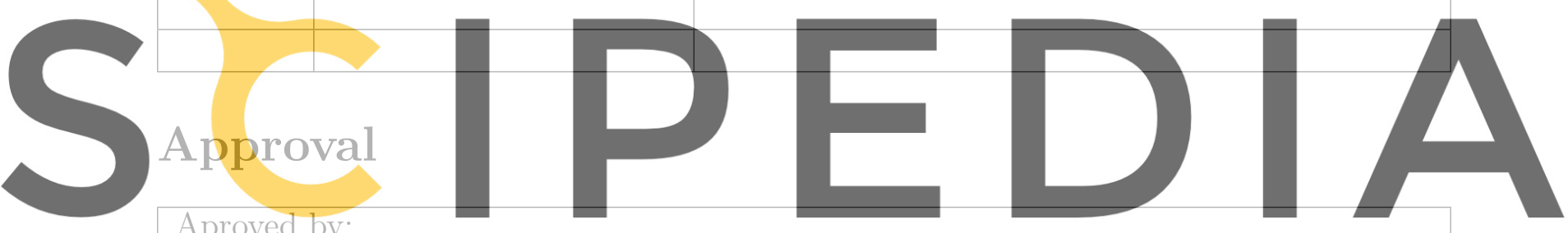

Aproved by:

Register for free at https/(www.scipedia.com to dortner

\begin{tabular}{|l|l|l|l|l|}
\hline WP leader & Riccardo Tosi & CIMNE & $30-05-19$ & OK \\
\hline Coordinator & Riccardo Rossi & CIMNE & $30-05-19$ & \\
\hline
\end{tabular}




\section{Executive summary}

This deliverable presents the software release of the Kratos Multiphysics software [3], "a framework for building parallel, multi-disciplinary simulation software, aiming at modularity, extensibility, and high performance. Kratos is written in $\mathrm{C}++$, and counts with an extensive Python interface". In this deliverable we focus on the development of Uncertainty Quantification inside Kratos. This takes place in the MultilevelMonteCarloApplication, a recent development inside the software that allows to deal with uncertainty quantification.
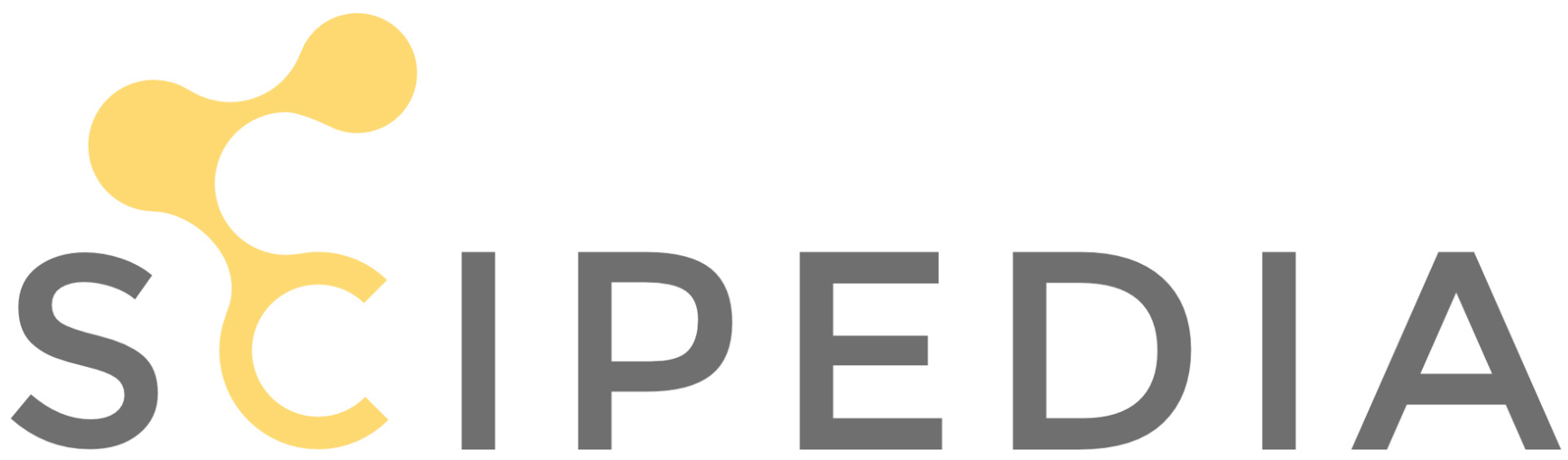

Register for free at https//www.scipedia.com to download the version without the watermark 


\section{Table of contents}

1 Introduction $\quad 6$

2 Methods $\quad 6$

2.1 Algorithms . . . . . . . . . . . . . . . . . . . 6

2.2 Adaptive refinement . . . . . . . . . . . . . . . . 6

2.3 High efficiency . . . . . . . . . . . . . . . . . . . . . . 6

2.4 Current results . . . . . . . . . . . . . . . . . . 6

3 Softwares
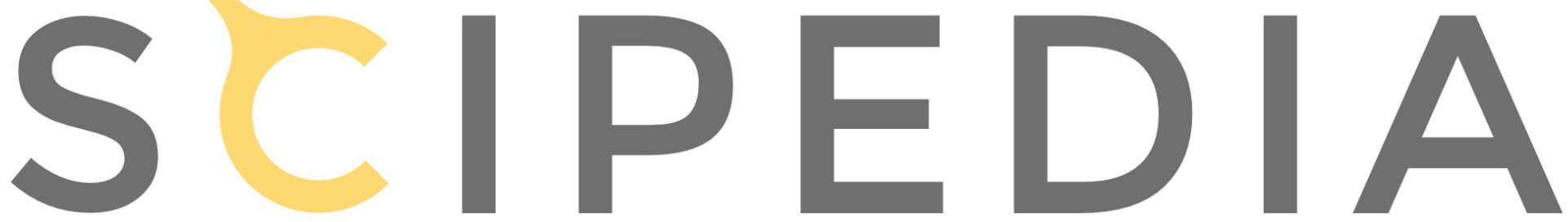

Register for free at https//www.scipedia.com to download the version without the watermark 


\section{Nomenclature / Acronym list}

\begin{tabular}{|l|l|}
\hline Acronym & Meaning \\
\hline Kratos & Kratos MultiPhysics \\
\hline MC & Monte Carlo \\
\hline MLMC & Multi Level Monte Carlo \\
\hline CMLMC & Continuation Multi Level Monte Carlo \\
\hline UQ & Uncertainty Quantification \\
\hline CFD & Computational Fluid Dynamics \\
\hline
\end{tabular}

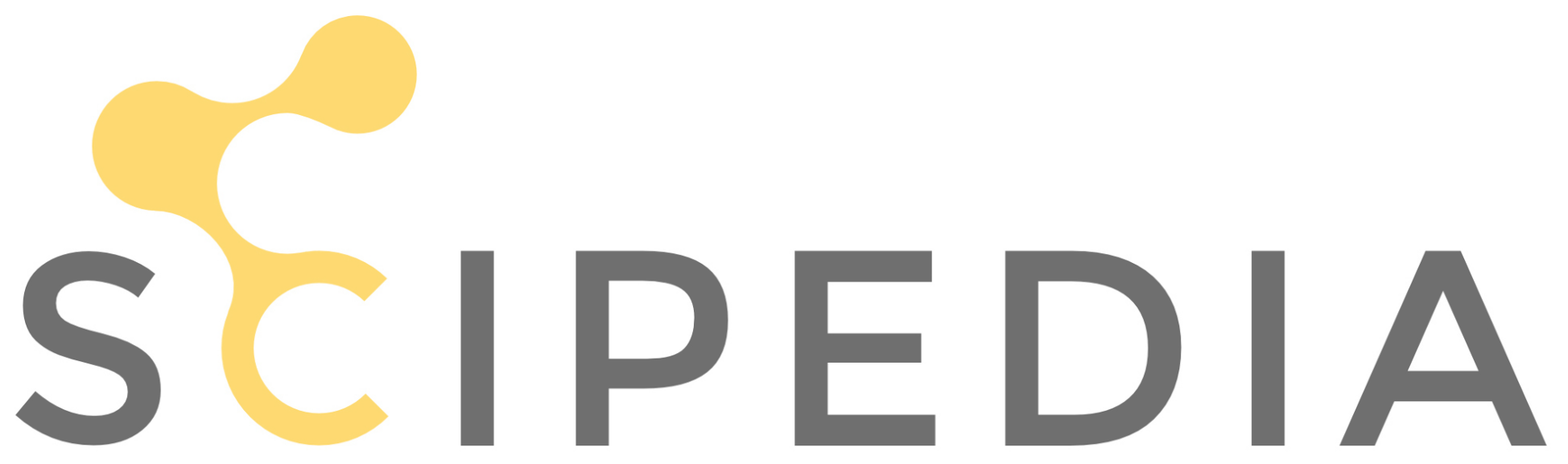

Register for free at https//www.scipedia.com to download the version without the watermark 


\section{Introduction}

The Kratos software is capable to deal with many different engineering fields, from CFD and convection diffusion to structural and solid mechanics problems. In all these fields it may be very important the necessity of dealing with uncertainties in some data, and to see how these affect the final results. The purpose of the Multi Level Monte Carlo application is exactly this: to study the uncertainty propagation in physical problems.

\section{Methods}

\subsection{Algorithms}

Different algorithms $[2,6,8]$ are presented in the software:

- Monte Carlo,

- Multi Level Monte Carlo,

- Continuation Multi Level Monte Carlo.

$\mathrm{MC}$ is the simplest

the other hand MLI

present an increasing

2.2

Adaptive refinement
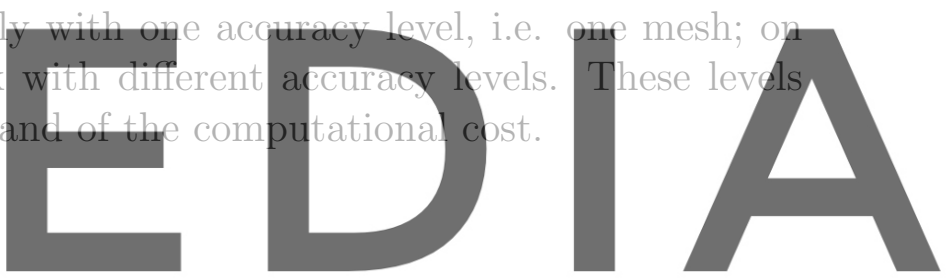

In the application this increasing accuracy is achieved through a solution-oriented space

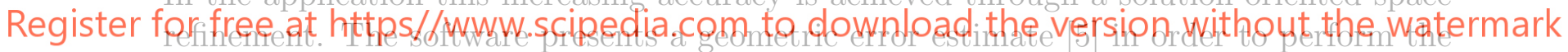

adaptive refinement. The metric is evaluated computing the hessian of the numerical solution, and this is given as input to the refinement software Mimg [4].

\subsection{High efficiency}

The necessity of running multiple simulations leads to the fundamental requirement of high efficiency. To achieve such result, there is a direct integration between Kratos and the chosen distributed software COMPSs $[1,7,9]$. The integration takes place at very high level, employing a Python layer. There is a direct integration between Kratos and PyCOMPSs, the COMPSs python library, and this allows to run many Kratos simulations independently and concurrently. A remarkable computational time efficiency is currently achieved.

\subsection{Current results}

The application presents a benchmark test case, that is a Poisson equation, and a more challenging engineering problem. This last studies the lift coefficient behavior of a compressible flow around an airfoil with random Mach number and random angle of attack. The application is currently capable to run in distributed environment, and has been tested that with 25 worker nodes (1200 cores) and 130000 simulations it fills all the hardware nodes. 


\section{Softwares}

A release of the Kratos software with DOI (10.5281/zenodo.3235261) can be found at the following link: https://zenodo.org/record/3235261.

The PyCOMPSs library can be found at this link: https://github.com/bsc-wdc/ compss.git.
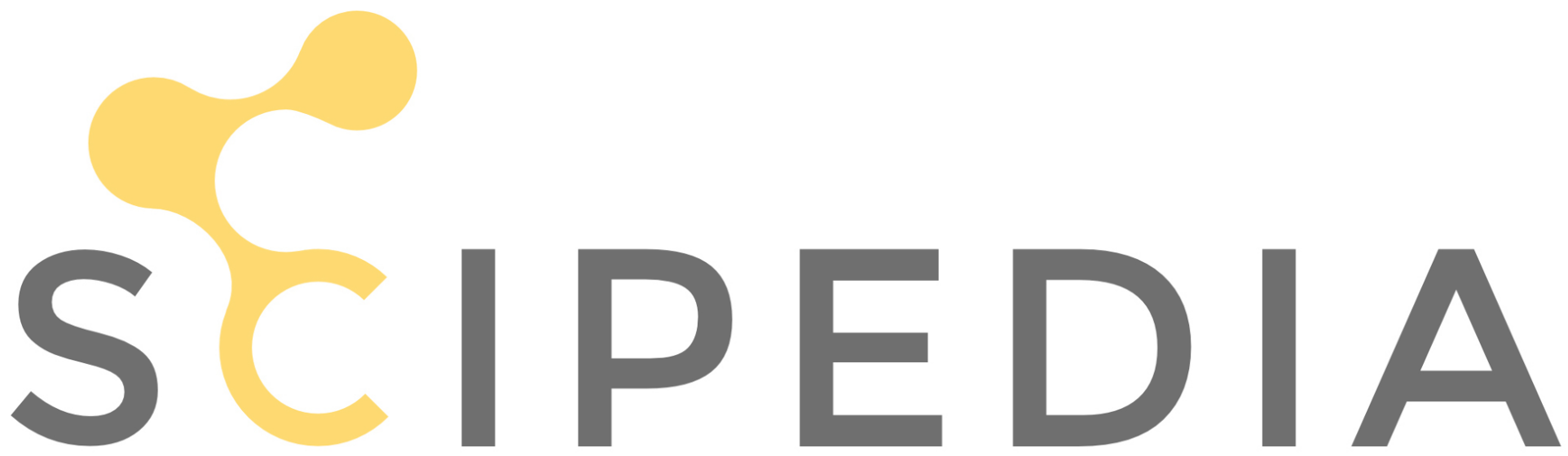

Register for free at https//www.scipedia.com to download the version without the watermark 


\section{References}

[1] R. Amela, C. Ramon-Cortes, J. Ejarque, J. Conejero, and R. M. Badia. Executing linear algebra kernels in heterogeneous distributed infrastructures with PyCOMPSs. Oil $\&$ Gas Science and Technology-Revue d'IFP Energies nouvelles, 73:47, 2018.

[2] N. Collier, A. L. Haji-Ali, F. Nobile, E. von Schwerin, and R. Tempone. A continuation multilevel Monte Carlo algorithm. BIT Numerical Mathematics, 55(2):399-432, 2015. ISSN 00063835. doi:10.1007/s10543-014-0511-3.

[3] P. Dadvand, R. Rossi, and E. Oñate. An object-oriented environment for developing finite element codes for multi-disciplinary applications. Archives of computational methods in engineering, 17(3):253-297, 2010.

[4] C. Dapogny, C. Dobrzynski, and P. Frey. Three-dimensional adaptive domain remeshing, implicit domain meshing, and applications to free and moving boundary problems. Journal of Computational Physics, 2014. ISSN 00219991. doi:10.1016/j.jcp.2014.01.005.

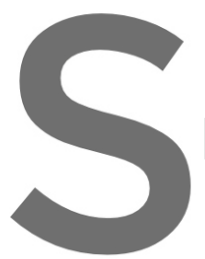

5] P. J. Frey and F. Alauzet. Anisotropic mesh adaptation for CFD computations. Computer Methods in Applied Mechanics and Engineering 194(48-49):5068-5082, 2005. ISSN 00457825. doi:10.1016/j.ema.2004.
M. B. Giles. Multilevel Monte Carl
56(3):607, 2008. ISSN 0030304X. UR.
df0f7244a429931ea175d.7d71e6591b6.

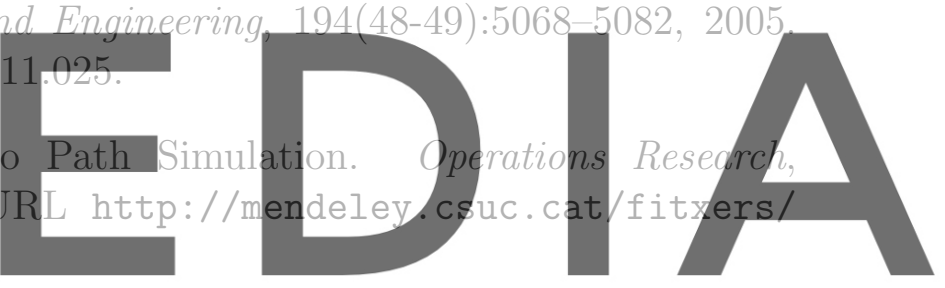

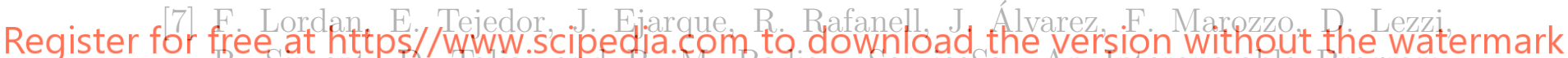
R. Sirvent, 15. Malia, and R. M. Badia. ServiceSs: An Interoperable Programming Framework for the Cloud. Journal of Grid Computing, 2014. ISSN 15707873. doi:10.1007/s10723-013-9272-5.

[8] M. Pisaroni, F. Nobile, and P. Leyland. A Continuation Multi Level Monte Carlo (CMLMC) method for uncertainty quantification in compressible inviscid aerodynamics. Computer Methods in Applied Mechanics and Engineering, 326:20-50, 2017.

[9] E. Tejedor, Y. Becerra, G. Alomar, A. Queralt, R. M. Badia, J. Torres, T. Cortes, and J. Labarta. PyCOMPSs: Parallel computational workflows in Python. International Journal of High Performance Computing Applications, 2017. ISSN 17412846. doi:10.1177/1094342015594678. 